PROCEEDINGS OF THE AMERICAN MATHEMATICAL SOCIETY

Volume 124, Number 10, October 1996

\title{
STABILITY OF SEMIGROUPS COMMUTING WITH A COMPACT OPERATOR
}

\author{
VŨ QUÔC PHÓNG
}

(Communicated by Palle E. T. Jorgensen)

\begin{abstract}
It is proved that if $T(t), S(t)$ are bounded $C_{0}$-semigroups on Banach spaces $X$ and $Y$, resp., and $C: Y \rightarrow X, K: Y \rightarrow Y$ are bounded operators with dense ranges such that $C$ intertwines $T(t)$ with $S(t)$ and $K$ commutes with $S(t)$, then $T(t)$ is strongly stable provided $A$ - the generator of $T(t)$ - does not have eigenvalue on $i \mathbf{R}$. An analogous result holds for powerbounded operators.
\end{abstract}

Below we denote by $T(t), t \geq 0$, a bounded, strongly continuous semigroup $\left(C_{0^{-}}\right.$ semigroup) of linear operators on a Banach space $X$, with generator $A$. We are concerned with the following stability problem: find conditions which imply that $T(t)$ is strongly stable, i.e. $\|T(t) x\| \rightarrow 0$ as $t \rightarrow \infty$, for all $x$ in $X$. In contrast to the case of finite dimensional space, when stability is equivalent to negativeness of the real parts of eigenvalues of $A$, there is evidently no simple characterization of strong stability for $C_{0}$-semigroups on Banach or Hilbert spaces. However, one can expect that such conditions (preferably in terms of the spectrum of $A$ ) can be found for particular classes of operators. Among known results which give conditions for strong stability, let us mention a theorem of Sz.-Nagy and Foias [5], that if $T(t)$ is a completely non-unitary contraction semigroup in a Hilbert space such that $m(i \sigma(A) \cap \mathbf{R})=0$, then $T(t)$ is strongly stable (where $m$ denotes the Lebesgue measure on $\mathbf{R}$ ). For semigroups on Banach spaces there has been obtained recently the following result, due to Arendt-Batty [1] and Lyubich and the author [4], [6] and now is sometimes known as the ABLP Theorem: If $\sigma(A) \cap i \mathbf{R}$ is countable and $P \sigma\left(A^{*}\right) \cap i \mathbf{R}$ is empty, where $P \sigma\left(A^{*}\right)$ is the point spectrum of $A^{*}$, then $T(t)$ is strongly stable. Results analogous to the Sz.-Nagy-Foias Theorem and the ABLP Theorem also hold for contractions in Hilbert space, and for powerbounded operators on Banach spaces, respectively.

The purpose of the present note is to show that the stability problem has a simple solution in the class of bounded $C_{0}$-semigroups which commute with a compact operator.

Theorem A. Assume that there is $t_{0}>0$ such that $T\left(t_{0}\right)$ commutes with a compact operator with dense range. Then $T(t)$ is strongly stable if (and only if) $P \sigma(A) \cap i \mathbf{R}=$ $\emptyset$.

Received by the editors April 17, 1995

1991 Mathematics Subject Classification. Primary 47D06.

(C)1996 American Mathematical Society 
Let $S(t)$ be another bounded $C_{0}$-semigroup on a Banach space $Y$, with generator $B$. We say that an operator $C: Y \rightarrow X$ intertwines $T(t)$ with $S(t)$ if $T(t) C=$ $C S(t), \forall t \geq 0$. Theorem A follows from the following more general result.

Theorem B. Assume that: (i) there exists an operator $C: Y \rightarrow X$ with dense range which intertwines $T(t)$ with $S(t)$, and (ii) there is $t_{0}>0$ such that $S\left(t_{0}\right)$ commutes with a compact operator $K$ with dense range. Then $T(t)$ is strongly stable if (and only if) $P \sigma(A) \cap i \mathbf{R}=\emptyset$.

Proof. The proof of the "only if" part is obvious. The proof of the "if" part is a simple application of the de Leeuw-Glicksberg Decomposition Theorem for almost periodic semigroups. Recall that a $C_{0}$-semigroup $T(t)$ is called almost periodic, if for every $x \in X$ the orbit $\mathcal{O}_{T}(x) \equiv\{T(t) x: t \geq 0\}$ is relatively compact. According to the de Leeuw-Glicksberg Decomposition Theorem [2] (see also [3]), if $T(t)$ is almost periodic, then $X=X_{s} \dot{+} X_{b}$, where $X_{s}=\left\{x \in X: \lim _{t \rightarrow \infty}\|T(t) x\|=0\right\}$, $X_{b}=\overline{\operatorname{span}}\left\{x \in X: \exists \lambda \in \mathbf{R}\right.$ such that $\left.T(t) x=e^{i \lambda t} x, \forall t \geq 0\right\}=\{x \in \mathcal{D}(A)$ : $\exists \lambda \in \mathbf{R}$ such that $A x=i \lambda x\}$. From this decomposition it follows that if the semigroup $T(t)$ is almost periodic and $P \sigma(A) \cap i \mathbf{R}=\emptyset$, then $T(t)$ is strongly stable.

Now since $T(t) C=C S(t), \forall t \geq 0$, and $S\left(t_{0}\right) K=K S\left(t_{0}\right)$, it follows that $T\left(t_{0}\right) C K=C K S\left(t_{0}\right)$, and hence $T\left(n t_{0}\right) C K=C K S\left(n t_{0}\right), \forall n=0,1,2, \ldots$. Since $C K$ is compact, it follows that $\left\{T\left(n t_{0}\right) x: n=0,1,2, \ldots\right\}$, and hence $\mathcal{O}_{T}(x)$ is relatively compact for every $x \in C K(Y)$. Since $C$ and $K$ have dense ranges, $C K(Y)$ is dense in $X$, and since $T(t)$ is a bounded semigroup, it follows that $\mathcal{O}_{T}(x)$ is relatively compact for each $x$ in $X$, i.e. $T(t)$ is almost periodic. Since $P \sigma(A) \cap i \mathbf{R}=\emptyset$, the preceding remark concerning the de Leeuw-Glicksberg Theorem implies that $T(t)$ is strongly stable.

Remarks. 1. If we do not assume that $C$ and $K$ have dense ranges, then from Theorem B it follows that the strong stability holds for the restrictions of the corresponding semigroup to the invariant subspace $\overline{(C K) Y}$.

2. It is easy to see that if $C: Y \rightarrow X$ intertwines $A$ with $B$, i.e. $C: \mathcal{D}(B) \rightarrow$ $\mathcal{D}(A)$, and $A C x=C B x, \forall x \in \mathcal{D}(B)$, then $C$ intertwines $T(t)$ with $S(t)$. Similarly, if $B$ commutes with $K$, i.e. $K: \mathcal{D}(A) \rightarrow \mathcal{D}(A)$, and $A K y=K A y, \forall y \in Y$, then $S(t)$ commutes with $K$.

Results analogous to Theorems A and B also hold for a single operator T. Recall that an operator $T$ is called strongly stable, if $\left\|T^{n} x\right\| \rightarrow 0$ as $n \rightarrow \infty, \forall x \in X$. Below $T$ and $S$ are power-bounded operators on Banach spaces $X$ and $Y$, respectively; $\mathbf{T}$ will denote the unit circle.

Theorem C. Assume that: (i) there exists an operator $C: Y \rightarrow X$ with dense range which intertwines $T$ with $S$, and (ii) $S$ commutes with some compact operator with dense range. Then $T$ is strongly stable if (and only if) $P \sigma(T) \cap i \mathbf{T}=\emptyset$. In particular, if $T$ commutes with a compact operator with dense range and $P \sigma(T) \cap$ $\mathbf{T}=\emptyset$, then $T$ is strongly stable.

\section{REFERENCES}

1. W. Arendt and C.J.K. Batty, Tauberian theorems and stability of one-parameter semigroups, Trans. Amer. Math. Soc. 306 (1988), 837-852. MR 89g:47053

2. K. de Leeuw and I. Glicksberg, Applications of almost periodic compactifications, Acta Math. 105 (1961), 63-97. MR 24:A1632 
3. Yu.I. Lyubich, Introduction to Banach Space Representations of Groups, Birkhäuser, Basel, 1988. MR 90i:22001

4. Yu.I. Lyubich and Vũ Quôc Phóng, Asymptotic stability of linear differential equations in Banach spaces, Studia Math. 88 (1988), 37-42. MR 89e:47062

5. B. Sz.-Nagy and C. Foias, Harmonic Analysis of Operators on Hilbert Space, North-Holland, Amsterdam, 1970. MR 43:947

6. Vũ Quôc Phóng and Yu.I. Lyubich, A spectral criterion for asymptotic almost periodicity of uniformly continuous representations of abelian semigroup, Teor. Funktsii, Funktsional. Anal. i Prilozhenia 50 (1988), 38-43; English transl., J. Soviet Math. 49 (1990), 1253-1266. MR 90e:22004

Department of Mathematics, Ohio University, Athens, Ohio 45701

E-mail address: qvu@bing.math.ohiou.edu 